\title{
Estudo da hemodinâmica venosa por meio da pletismografia a ar no pré e pós-operatório de varizes dos membros inferiores
}

\author{
Venous hemodynamic study by air plethysmography in the pre-and \\ postoperative period of lower limb varicose veins \\ Nei Rodrigues Alves Dezotti ${ }^{1}$, Edwaldo Edner Joviliano ${ }^{1}$, Mariana Key Toma ${ }^{2}$, Takachi Moriya ${ }^{3}$, \\ Carlos Eli Piccinato ${ }^{4}$
}

\section{Resumo}

Contexto: O tratamento cirúrgico das varizes primárias dos membros inferiores deve proporcionar alívio da estase venosa, a fim de evitar a evolução da doença venosa para estágios de maior gravidade clínica.

Objetivo: Estudar as alterações da hemodinâmica venosa em pacientes portadores de varizes primárias dos membros inferiores no pré e pós-operatório, utilizando a pletismografia a ar.

Método: Foram estudados 63 membros inferiores em 39 pacientes (35 mulheres e quatro homens), com média de idade igual a 46,3 anos, operados no Hospital das Clínicas da Faculdade de Medicina de Ribeirão Preto da Universidade de São Paulo, no período de janeiro de 2001 a dezembro de 2004. Os membros inferiores foram classificados de acordo com a classificação CEAP, em critério clínico $=\mathrm{C}_{2}$ a $\mathrm{C}_{6}\left(\mathrm{C}_{2}\right.$ $=6, \mathrm{C}_{3}=32, \mathrm{C}_{4}=15, \mathrm{C}_{5}=7$ e $\mathrm{C}_{6}=3$ ), critério etiológico $=\mathrm{E}_{\mathrm{p}}$, critério anatômico $=\mathrm{A}_{\mathrm{s}}$ e critério fisiopatológico $=\mathrm{P}_{\mathrm{r}}$. Os pacientes foram avaliados por exame clínico pré e pós-operatório, mapeamento dúplex pré-operatório e pletismografia a ar pré e pós-operatória.

Resultados: Houve melhora da hemodinâmica venosa no pós-operatório, demonstrada pela diminuição do índice de enchimento venoso e da fração de volume residual e aumento da fração de ejeção nos membros inferiores submetidos à operação de varizes.

Conclusão: A operação venosa superficial aliviou a estase venosa e proporcionou adequado tratamento, com o objetivo de interromper a evolução fisiopatológica da doença venosa crônica, em qualquer nível de gravidade clínica.

Palavras-chave: Varizes, membros inferiores, pletismografia.

\begin{abstract}
Background: Surgical treatment of primary varicose veins of the lower limbs might contribute to venous stasis relief by preventing evolution of the venous disease to worse severity stages.

Objective: To study venous hemodynamic changes in patients with primary varicose veins of the lower limbs during the pre- and postoperative period using air plethysmography.

Method: Sixty-three lower limbs of 39 patients (35 females and four males, mean age of 46.3 years) were evaluated. They were all operated at Hospital das Clínicas, Faculdade de Medicina de Ribeirão Preto, Universidade de São Paulo, between January 2001 and December 2004. Lower limbs were classified according to the CEAP classification: clinical criteria $=\mathrm{C} 2$ to $\mathrm{C} 6(\mathrm{C} 2=6, \mathrm{C} 3=32, \mathrm{C} 4=15$, $\mathrm{C} 5=7$ and $\mathrm{C} 6=3$ ), etiologic criteria $=\mathrm{Ep}$, anatomic criteria $=\mathrm{As}$, and pathophysiological criteria $=\operatorname{Pr}$. The patients were submitted to pre- and postoperative clinical examination, preoperative duplex scan and pre- and postoperative air plethysmography.

Results: Improvement in venous hemodynamics was observed after surgery, confirmed by reduction in the venous filling index and residual volume fraction and increase in the ejection fraction in the lower limbs submitted to varicose vein surgery.

Conclusion: Superficial varicose vein stripping contributed to venous stasis relief and provided appropriate treatment, preventing pathophysiological evolution of chronic venous disease independently of clinical severity.
\end{abstract}

Keywords: Varicose veins, lower extremity, plethysmography.

1. Doutor. Médico assistente, Divisão de Cirurgia Vascular e Endovascular, Departamento de Cirurgia e Anatomia, Faculdade de Medicina de Ribeirão Preto Universidade de São Paulo (FMRP-USP), Ribeirão Preto, SP.

2. Ex-monitora, Divisão de Cirurgia Vascular e Endovascular, Departamento de Cirurgia e Anatomia, FMRP-USP, Ribeirão Preto, SP.

3. Doutor. Professor, Divisão de Cirurgia Vascular e Endovascular, Departamento de Cirurgia e Anatomia, FMRP-USP, Ribeirão Preto, SP.

4 . Professor titular, Divisão de Cirurgia Vascular e Endovascular, Departamento de Cirurgia e FMRP-USP, Ribeirão Preto, SP.

Este trabalho é parte da tese de doutorado apresentada ao Departamento de Cirurgia e Anatomia, no Programa de Clínica Cirúrgica, Faculdade de Medicina de Ribeirão Preto - Universidade de São Paulo (USP), Ribeirão Preto, SP.

Não foram declarados conflitos de interesse associados à publicação deste artigo.

Artigo submetido em 09.07.08, aceito em 26.12.08.

J Vasc Bras. 2009;8(1):21-28.

Copyright $\odot 2009$ by Sociedade Brasileira de Angiologia e de Cirurgia Vascular 


\section{Introdução}

As varizes primárias dos membros inferiores (MMII) representam uma das afeç̧ões mais frequentes nos pacientes que procuram atendimento em ambulatórios de cirurgia vascular, gerando uma demanda para tratamento que, muitas vezes, supera a capacidade de atendimento do sistema público de saúde, com consequente formação de filas em diversas cidades do nosso país ${ }^{1}$.

As varizes ou veias varicosas dos MMII, categorizadas como $\mathrm{C}_{2}$ na classificação internacional $\mathrm{CEAP}^{2}$, são conceituadas como veias dilatadas, tortuosas e alongadas, com alterações de sua função ${ }^{3}$. São mais comuns no sexo feminino, estando associadas também a outros fatores, como idade, raça, número de gestações, ortostatismo prolongado, obesidade e função intestinal ${ }^{3-9}$.

As varizes podem ser primárias ou essenciais, quando o sistema venoso profundo está normal, e secundárias, em consequência de doença no sistema venoso profundo, como refluxo e/ou obstrução ${ }^{3,7}$.

As queixas que motivam a consulta médica são diversas, tais como: problemas estéticos, dor, edema, sensação de peso nos MMII, cãibras e prurido ${ }^{5,6,10-12}$.

A pletismografia a ar (PGA) é um método nãoinvasivo que, no início dos anos 60 , começou a ser usado para estudar mudanças relativas do volume nos MMII em resposta a alterações posturais e aos exercícios musculares ${ }^{13}$.

A PGA foi introduzida por Christopoulos et al., em 1988, como um teste não-invasivo para avaliação quantitativa da doença venosa crônica (DVC). Esses autores descreveram, em seu trabalho, que o índice de enchimento venoso (IEV) mensura os diversos graus de refluxo venoso, sendo, assim, um instrumento importante para o diagnóstico da DVC, em situações onde os sinais clínicos não são evidentes ${ }^{14}$.

Além da análise do refluxo venoso, apresentado como ponto principal resultante do refluxo valvar, a PGA também avalia a função da bomba muscular da panturrilha pela medida da fração de ejeção (FE) e, ainda, da fração de volume residual (FVR) ${ }^{15}$.

Pode-se estender essas avaliações para analisar os efeitos do tratamento clínico ou cirúrgico das varizes primárias dos MMII, que objetivam melhorar o refluxo venoso e a função de bomba muscular da panturrilha.

Assim, planejou-se estudar quantitativamente as alterações da hemodinâmica venosa em pacientes portadores de varizes de MMII no período pré-operatório e comparar com os resultados observados no pósoperatório, com o objetivo de investigar o possível alívio da estase venosa após a ressecção da safena magna, veias varicosas e ligadura de perfurantes incompetentes utilizando-se a PGA.

O objetivo deste trabalho é estudar quantitativamente as alterações hemodinâmicas venosas por meio da PGA no pré e pós-operatório de varizes dos MMII.

\section{Casuística e método}

Foram avaliados, prospectivamente, 39 pacientes (63 MMII), sendo 35 (88,6\%) mulheres e quatro $(11,4 \%)$ homens, com idade média de 46,3 anos, atendidos no Ambulatório de Cirurgia Vascular do Hospital das Clínicas (HC) da Faculdade de Medicina de Ribeirão Preto (FMRP) da Universidade de São Paulo (USP) e operados por um único docente da disciplina de Cirurgia Vascular, no período de janeiro de 2001 a dezembro de 2004. Os pacientes foram selecionados de acordo com os seguintes critérios:

- Critérios de exclusão: pacientes com história pregressa de operação de varizes, pacientes portadores de malformações congênitas, diabetes melito, cardiopatias, vasculopatias arteriais e linfáticas, colagenoses e miopatias, traumatismos com ou sem fraturas associadas, síndromes pós-flebíticas, pacientes portadores de osteopatias e artropatias isoladas ou associadas e aqueles que não assinaram o termo de consentimento.

- Critérios de inclusão: pacientes portadores de varizes primárias sintomáticas dos MMII, categorizados de acordo com a classificação internacional CEAP, sendo: critério clínico $=\mathrm{C}_{2} a_{6}$; critério etiológico $=\mathrm{E}_{\mathrm{p}}$; critério anatômico $=\mathrm{A}_{\mathrm{s}}$; e critério fisiopatológico $=\mathrm{P}_{\mathrm{r}}{ }^{2}$.

Foram estudados 63 MMII, sendo: $\mathrm{C}_{2}=6(9,5 \%), \mathrm{C}_{3}=$ $32(50,8 \%), \mathrm{C}_{4}=15(23,8 \%), \mathrm{C}_{5}=7(11,1 \%)$ e $\mathrm{C}_{6}=3$ $(4,8 \%)$. 
Os MMII foram avaliados por exame clínico pré e pós-operatório, mapeamento dúplex (MD) préoperatório e PGA pré e pós-operatória (30 a 40 dias após a operação).

\section{Exame clínico}

O paciente foi colocado em ambiente iluminado e examinado em posição ortostática, permitindo, assim, o enchimento e visualização das veias varicosas superficiais. Foram avaliadas as faces medial, lateral anterior e posterior dos MMII.

O exame foi realizado segundo os padrões clássicos semiológicos, através da inspeção, palpação, percussão e ausculta, além da realização da prova do garrote (teste de Brodie-Trendelenburg).

\section{Mapeamento dúplex}

O MD foi feito com o objetivo de excluir pacientes portadores de doenças do sistema venoso profundo.

Foram utilizadas sondas lineares de alta frequência (7,0 a $10 \mathrm{MHz}$ ), capazes de produzir imagens em modo B, Doppler colorido e curvas espectrais a partir do Doppler pulsado. O aparelho utilizado foi o Aspen-Siemens ${ }^{\circledR}$ (Erlangen, Alemanha).

Considerou-se insuficiente o vaso que apresentasse refluxo com duração maior que 0,5 segundo.

Os exames foram realizados por um único examinador independente, e o resultado foi informado ao investigador principal.

\section{Pletismografia a ar}

Os exames foram realizados no Laboratório de Investigação Vascular Não-Invasiva da Divisão de Cirurgia Vascular e Endovascular do HC-FMRP-USP, no período vespertino, por dois examinadores, concomitantemente. As manobras foram iniciadas somente após demonstração do exame ao paciente e confiança em sua compreensão.

Os exames foram realizados no pré-operatório e entre o trigésimo e quadragésimo quinto dia do pós-operatório.

A função da bomba muscular da panturrilha foi estudada pela PGA. O aparelho utilizado foi o SDV 3000 da marca Angiotec ${ }^{\circledR}$ (Belo Horizonte, Brasil), com calibração automatizada por computador. Utilizou-se, para o estudo, a técnica descrita e padronizada por Christopoulos et al., em 1988, e os parâmetros avaliados foram: IEV, FE e FVR ${ }^{14}$.

O IEV é a variação de volume produzida no membro dividida pelo tempo correspondente em segundos, na passagem da posição deitada para a posição ortostática, calculado mediante a fórmula IEV $=90 \% \mathrm{x}$ volume venoso (VV) / tempo de $90 \%$ do enchimento venoso (TEV90) e expresso em mL/s.

Estudou-se a função de bomba da panturrilha por meio dos dados obtidos pelas variáveis FE e FVR.

A FE fornece uma estimativa do retorno venoso durante a atividade física e de alterações que resultem em menos sangue ejetado. A FE é a porcentagem do VV total ejetado em uma única contração da musculatura da panturrilha, sendo calculada pela fórmula $\mathrm{FE}=$ volume ejetado (VE) / VV x 100.

A FVR correlaciona-se linearmente com a pressão venosa ambulatorial (PVA), medindo-a de forma nãoinvasiva, ou seja, pela fórmula $\mathrm{FVR}=$ volume residual (VR) / VV x $100^{16}$.

Os valores de literatura considerados normais são IEV $<2, \mathrm{FE}>40 \%$ e FVR $<35 \% 14,17,18$.

\section{Tratamento cirúrgico}

O tratamento cirúrgico foi realizado objetivando-se a correção dos pontos de refluxo nos membros estudados.

Dos 63 MMII estudados, fez-se safenectomia magna radical (SMR) e exérese de varizes (EV) em 55 e SMR com ligadura de perfurantes (LP) e EV em oito.

\section{Análise estatística}

As variáveis estudadas por meio da PGA para avaliação hemodinâmica no pré e no pós-operatório foram o IEV, a FE e a FVR. Essas variáveis não apresentaram distribuição de normalidade pelo teste de KolmogorovSmirnov $(\mathrm{p}<0,01)$. Portanto, utilizou-se a abordagem não-paramétrica na análise, adotando-se como nível de significância $\mathrm{p} \leq 0,05$.

Compararam-se os valores pré e pós-operatórios das variáveis IEV, FVR e FE, obtidas pela PGA em 63 
Tabela 1 - Comparação entre valores pré e pós-operatórios do índice de enchimento venoso em $\mathrm{mL} / \mathrm{s}$

\begin{tabular}{lcccccc}
\hline IEV & $\mathbf{n}$ & $\mathbf{1}^{\mathbf{0}}$ quartil (P25) & Mediana (P50) & $\mathbf{3}^{\mathbf{0}}$ quartil (P75) & Média & Desvio padrão \\
\hline Pré & 63 & 2,47 & 3,72 & 5,38 & 4,11 & 2,28 \\
Pós & 63 & 1,15 & 1,77 & 2,60 & 2,17 & 1,40 \\
\hline
\end{tabular}

IEV = índice de enchimento venoso; $\mathrm{n}=$ número de membros inferiores. $\mathrm{Z}=5,71$; valores de IEV $=\mathrm{mL} / \mathrm{s} ; \mathrm{p}<0,001$.

MMII, utilizando-se o teste não-paramétrico de Wilcoxon para amostras pareadas representadas em tabelas. Utilizaram-se, ainda, gráficos formados por caixas com três linhas horizontais e uma linha vertical. A linha horizontal intermediária representa a mediana, valor abaixo do qual estão $50 \%$ das observações. A linha inferior é o primeiro quartil, e a superior, o terceiro quartil, com valores que deixam 25 e $75 \%$ dos dados abaixo deles, respectivamente ${ }^{19}$. Observam-se "asteriscos" na linha vertical, que representam os valores discrepantes.

Todos os pacientes receberam informações claras e objetivas dentro de sua compreensão para esclarecer a finalidade, propósitos, riscos e benefícios da pesquisa aplicada. Foi preparado um termo de consentimento livre e esclarecido, aprovado pelo Comitê de Ética em Pesquisa do HC-FMRP-USP, atendendo às diretrizes e normas regulamentadoras de pesquisa envolvendo seres humanos, contidas na resolução ${ }^{\circ} 196$, de 10 de outubro de 1996, e na resolução $\mathrm{n}^{\circ} 251$, de 7 de agosto de 1997, do Conselho Nacional de Saúde, bem como na Declaração de Helsinki VI e Código de Nuremberg.

\section{Resultados}

Foi feita a comparação entre os valores pré e pósoperatórios das variáveis IEV, FVR e FE obtidas pela PGA em 63 MMII.

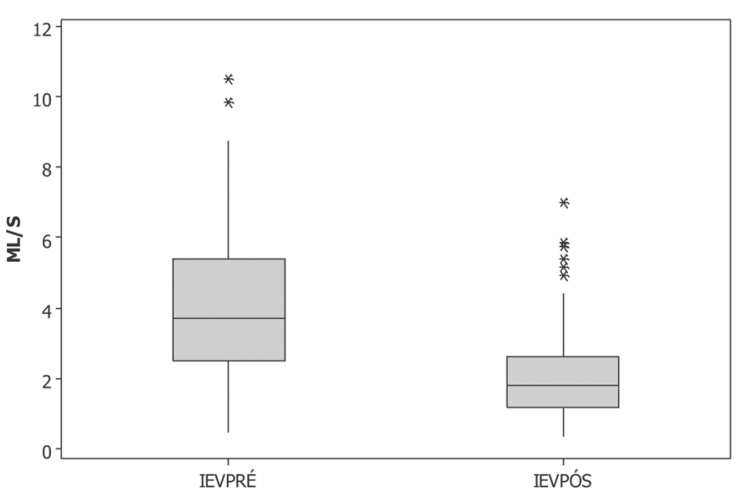

Figura 1 - Representação gráfica do índice de enchimento venoso no pré e pós-operatório (mediana e quartis)

Foram comparados os valores da mediana, dos quartis e da média das variáveis IEV no pré e pós-operatório, conforme Tabela 1.

Houve queda significativa dos valores do IEV no pósoperatório, quando comparados com os do préoperatório (Figura 1).

Os valores da mediana, dos quartis e a média da FE no pré e pós-operatório estão representados na Tabela 2. Comparando-se os valores da FE no pré e pósoperatório, observa-se que houve elevação significativa da FE no pós-operatório (Figura 2).

Tabela 2 - Comparação entre valores pré e pós-operatórios da fração de ejeção em \%

\begin{tabular}{lcccccc}
\hline FE & $\mathbf{N}$ & $\mathbf{1}^{\mathbf{0}}$ quartil (P25) & Mediana (P50) & $\mathbf{3}^{\mathbf{0}}$ quartil (P75) & Média & Desvio padrão \\
\hline Pré & 63 & 26,00 & 38,40 & 57,10 & 41,89 & 19,92 \\
Pós & 63 & 38,10 & 49,10 & 75,80 & 54,82 & 22,94 \\
\hline
\end{tabular}

$\mathrm{FE}=$ fração de ejeção; $\mathrm{n}=$ número de membros inferiores.

$\mathrm{Z}=4,10$; valores de $\mathrm{FE}=\% ; \mathrm{p}<0,001$. 


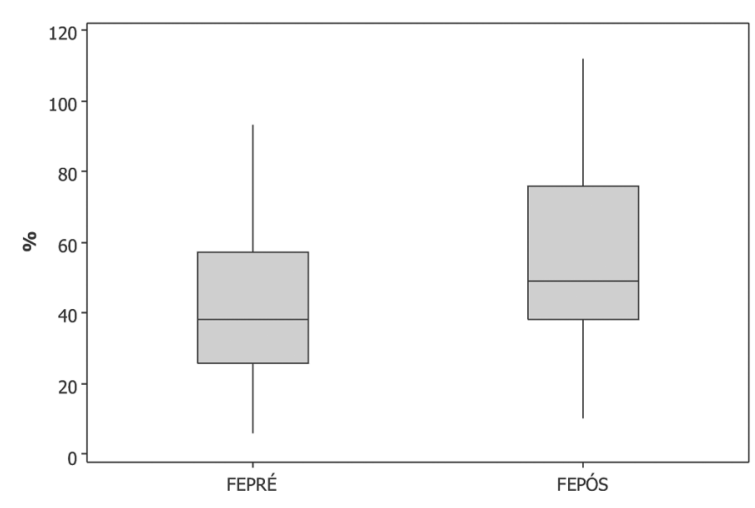

Figura 2 - Representação gráfica da fração de ejeção no pré e pós-operatório (mediana e quartis)

Os valores da mediana, dos quartis e da média da FVR no pré e pós-operatório estão representados na Tabela 3. Houve queda significativa dos valores da FVR no pós-operatório em relação aos valores do préoperatório (Figura 3).

Exames não-invasivos têm contribuído significativamente para a compreensão das alterações venosas que ocorrem na DVC.

O MD tem sido utilizado como substituto da flebografia na identificação do refluxo venoso produzido pela incompetência valvar de veias profundas, superficiais ou perfurantes dos $\mathrm{MMII}^{20}$. Essa técnica permite ao examinador determinar as alterações anatômicas que contribuem para DVC e obter informações a respeito do impacto das mesmas sobre a hemodinâmica venosa. É capaz de identificar locais onde há alterações patológicas, mas a análise quantitativa das alterações hemodinâmicas desses locais depende muito da habilidade técnica e experiência do examinador, além de ser necessário tempo prolongado para a realização do exame.

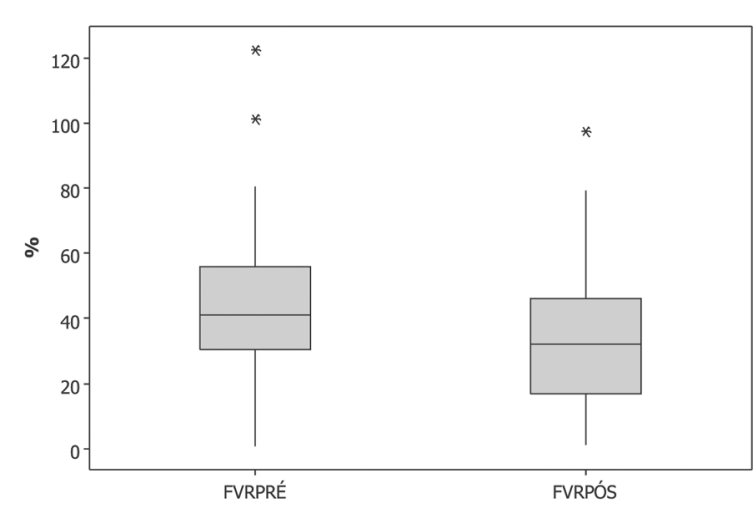

Figura 3 - Representação gráfica da fração de volume residual no pré e pós-operatório (mediana e quartis)

A fotopletismografia (FPG) tem sido utilizada como exame de seleção em muitos laboratórios, pois o tempo de reenchimento detectado pela FPG, que corresponde ao IEV medido pela PGA, tem-se mostrado sensível, embora não específico, como indicador de refluxo. Alguns autores demonstraram que o IEV fornecido pela FPG pode detectar se a incompetência é do sistema venoso superficial ou profundo ${ }^{21,22}$; porém, em trabalho realizado em nosso país por Sardinha, em 1987, não se obteve resultado semelhante ${ }^{23,24}$. A desvantagem da FPG é o fato de não identificar diferentes graus de gravidade clínica em relação à insuficiência venosa ${ }^{25}$.

A PGA mede vários parâmetros da função venosa dos MMII, incluindo o IEV, FE e FVR.

O IEV corresponde ao enchimento venoso do membro estudado como resultado da mudança do mesmo da posição supina para a ortostática e representa a medida do refluxo venoso ${ }^{14}$. Foi considerado o melhor parâmetro, obtido pela PGA, indicador da gravidade clínica do membro estudado ${ }^{14,26,27}$.

Tabela 3 - Comparação entre valores pré e pós-operatórios da fração de volume residual em \%

\begin{tabular}{lcccccc}
\hline FVR & $\mathbf{n}$ & $\mathbf{1}^{\mathbf{0}}$ quartil (P25) & Mediana (P50) & $\mathbf{3}^{\mathbf{0}}$ quartil (P75) & Média & Desvio padrão \\
\hline Pré & 63 & 30,50 & 41,00 & 56,10 & 43,10 & 21,48 \\
Pós & 63 & 17,00 & 32,30 & 46,40 & 33,78 & 20,09
\end{tabular}

$\mathrm{FVR}=$ fração de volume residual; $\mathrm{n}=$ número de membros inferiores.

$\mathrm{Z}=2,75$; valores de $\mathrm{FVR}=\% ; \mathrm{p}<0,001$. 
A FE, por ser a razão entre o VE e o VV, tem seus índices de variação independentes do tamanho do membro estudado. Dessa forma, pode ser utilizada na comparação de diferentes grupos de pacientes, sem haver a necessidade de medir o VV de cada membro estudado. Fornece uma ideia do retorno venoso durante a atividade física e também das alterações que resultam em menos sangue ejetado. A FE diminui na doença venosa, principalmente em situações de oclusão de sistema venoso profundo e quando há fatores que determinem uma diminuição da função de bomba da panturrilha ${ }^{17}$.

Uma FE de 30 a 70\% é observada em MMII com varizes primárias e pode cair para valores próximos a $10 \%$ na doença venosa profunda ${ }^{28}$.

A medida do VR e da FVR mostra que, na vigência de uma insuficiência venosa nos MMII, há diminuição do esvaziamento venoso e, consequentemente, do VE. Após um movimento de flexão do pé como resultado da contração muscular da panturrilha, o volume de sangue expelido pelas veias da perna corresponde ao sangue proveniente dos capilares associado ao do refluxo venoso em cada período de relaxamento. Se houver insuficiência do sistema venoso no membro estudado, haverá, nessa fase, aumento significativo da FVR, em contraste com a medida do VE e da FE, que não dependem do refluxo venoso.

Foi demonstrada a existência de uma correlação linear entre a PVA e a FVR, indicando que uma estimativa da PVA pode ser obtida não-invasivamente pela FVR utilizando-se a PGA ${ }^{22,29}$.

Embora nem todos os autores concordem, estabeleceu-se que, quanto maior a PVA, de modo proporcional e linear, maior é a FVR, sendo que uma FVR de $35 \%$ corresponde a aproximadamente $35 \mathrm{mmHg}$ de PVA, por exemplo. Valores inferiores a $35 \%$ são considerados adequados. Há uma relação direta entre a pressão venosa e a gravidade da doença venosa ${ }^{30}$.

Muitos estudos quantificaram o refluxo venoso obtido pela PGA e demonstraram que os parâmetros obtidos podem ser correlacionados aos estágios clínicos e também ser utilizados para monitorizar os resultados da operação venosa após diferentes tipos de intervenção ${ }^{31-37}$.
A importância do refluxo venoso superficial em estágios avançados da DVC foi objeto de estudo de outros autores, que concluíram que mais de $50 \%$ de seus pacientes com úlcera flebopática tinham apenas insuficiência venosa superficial $^{38}$.

Planejou-se este estudo com o objetivo de avaliar as alterações hemodinâmicas após operação venosa em pacientes portadores de insuficiência venosa superficial com sistema venoso profundo competente.

Avaliaram-se quantitativamente as alterações hemodinâmicas de MMII tratados cirurgicamente de varizes por meio da PGA, estudando-se os valores do IEV, da FE e da FVR no pré e no pós-operatório. Houve melhora significante dos parâmetros estudados após o tratamento, demonstrada pela diminuição do IEV e da FVR e aumento da FE.

A diminuição do IEV pode ser explicada pela diminuição do refluxo venoso após a EV. Possíveis explicações para a não-redução do IEV pós-operatório em alguns membros podem ser a existência de capilares dilatados na porção mais distal da perna, formando pequenas fístulas arteriovenosas em pacientes com $\mathrm{DVC}^{31} \mathrm{ou}$ falha no tratamento cirúrgico. A redução da FVR e o aumento da FE no pós-operatório resultam da diminuição do refluxo venoso, avaliado pelo IEV, e consequente diminuição do VV do membro inferior estudado. Deve-se lembrar que o VV do membro refere-se ao volume de sangue presente nas veias e varia de acordo com a posição do membro, as atividades da bomba muscular e a integridade das valvas venosas ${ }^{17}$.

Sabe-se que a DVC agrava-se progressivamente, podendo ter consequências maiores no futuro. É possível contribuir para o atendimento e tratamento mais racional dos pacientes portadores de doenças venosas dos MMII utilizando-se exames não-invasivos, com o objetivo de diminuir o impacto socioeconômico provocado por essas doenças.

Sugere-se, com base nessa investigação, adotar a PGA associada ao exame clínico e MD como protocolo na avaliação da doença venosa no pré e pós-operatório, pois a PGA é um exame não-invasivo, de baixo custo, fácil execução, e seus resultados quantificam hemodinamicamente a doença venosa. 
Conclui-se, neste trabalho, que a operação venosa superficial é uma forma adequada de tratamento para tentar interromper a evolução fisiopatológica em qualquer nível de gravidade clínica (CEAP) da DVC.

\section{Referências}

1. Souza E, Suzuki RT, Veloso MV. Estudos dos fatores de risco nos pacientes portadores de varizes dos membros inferiores em serviço público de saúde. Rev Ang Cir Vasc. 2005;4:161-4.

2. Porter JM, Moneta GL. Reporting standards in venous disease: an update. International Consensus Committee on Chronic Venous Disease. J Vasc Surg. 1995;21:635-45.

3. Maffei FH. Varizes dos membros inferiores: epidemiologia, etiopatogenia e fisiopatologia. In: Maffei FH, Lastoria S, Yoshida WB, Rollo HA. Doenças vasculares periféricas. $3^{\mathrm{a}}$ ed. Rio de Janeiro: Medsi; 2002. p. 1499.

4. Maffei FH, Magaldi C, Pinho SZ, et al. Varicose veins and chronic venous insufficiency in Brazil: prevalence among 1755 inhabitants of a country town. Int J Epidemiol. 1986;15:210-7.

5. Carpentier P, Priollet P. [Epidemiology of chronic venous insufficiency]. Presse Med. 1994;23:197-201.

6. Callam MJ. Epidemiology of varicose veins. Br J Surg. 1994;81:167-73.

7. Belcaro G, Nicolaides AN, Veller M. Varicoses veins. In: Venous disorders: a manual of diagnosis and treatment. London: WB Saunders; 1995. p. 52-68.

8. Fowkes FG, Evans CJ, Lee AJ. Prevalence and risk factors to chronic venous insufficiency. Angiology. 2001;52: Suppl 1:S5-15.

9. Cesarone MR, Belcaro G, Nicolaides AN, et al. 'Real' epidemiology of varicose veins and chronic venous diseases: the San Valentino vascular screening project. Angiology. 2002;53:119-30.

10. Becker F. [Mechanisms, epidemiology and clinical evaluation of venous insufficiency of the lower limbs]. Rev Prat. 1994;44:726-31.

11. Belcaro G, Nicolaides AN, Veller M. Assessment of the venous and lymphatic systems. In: Venous disorders: a manual of diagnosis and treatment. London: WB Saunders; 1995. p. 41.

12. Garrido M, Fonseca Filho VL. Exame do paciente varicoso. In: Maffei, FH. Doenças vasculares periféricas. $3^{\mathrm{a}}$ ed. Rio de Janeiro: Medsi; 2002. p. 1521.

13. Sacchi AA, Castro AA, Pitta GB, Miranda Jr F. Avaliação da bomba muscular da panturrilha em pacientes portadores de varizes primárias dos membros inferiores através da pletismografia a ar. J Vasc Bras. 2007;6:25-34.

14. Christopoulos D, Nicolaides AN, Szendro G. Venous reflux: quantification and correlation with the clinical severity of chronic venous disease. Br J Surg. 1988;75:352-6.

15. Christopoulos D, Nicolaides AN, Cook A, Irvine A, Galloway JM, Wilkinson A. Pathogenesis of venous ulceration in relation to the calf muscle pump function. Surgery. 1989;106:829-35.

16. Criado E. Laboratory evaluation of patient with chronic venous insufficiency. In: Rutherford RB. Vascular surgery. $4^{\mathrm{a}}$ ed. Philadelphia: WB Saunders; 1995. p. 1771-85.
17. Evangelista SS. Pletismografia no estudo das doenças venosas. In: Maffei FH. Doenças vasculares periféricas. $3^{\mathrm{a}}$ ed. Rio de Janeiro: Medsi; 2002. p. 479.

18. Figueiredo MA. Avaliação do efeito da meia elástica na hemodinâmica venosa dos membros inferiores de pacientes com insuficiência venosa crônica. J Vasc Bras. 2004;3:231-7.

19. Conover WJ. Practical nonparametric statistics. 2nd ed. New York: John Wiley \& Sons; 1980.

20. Szendro G, Nicolaides AN, Zukowski AJ, et al. Duplex scanning in the assessment of deep venous incompetence. $J$ Vasc Surg. 1986;4:237-42.

21. Abramowitz HB, Queral LA, Finn WR, et al. The use of photoplethysmography in the assessment of venous insufficiency: a comparison to venous pressure measurements. Surgery. 1979;86:434-41.

22. Belcaro G, Labropoulos N, Christopoulos D, et al. Noninvasive tests in venous insufficiency. J Cardiovasc Surg (Torino). 1993;34:3-11.

23. Sardinha WE, Miranda Jr. F, Francisco Jr. J, Pitta GB, Burihan E. Avaliação da insuficiência venosa crônica pela fotopletismografia. Cir Vasc Ang. 1988;4:7-11.

24. Miranda Júnior F, Burihan E. Photoplethysmography vs ascending and descending phlebography in chronic venous insufficiency. Medicographia. 1989;11:15-7.

25. Bays RA, Healy HA, Atnip RG, Neumyer M, Thiele BL. Validation of air plethysmography, photoplethysmography, and duplex ultrasonography in the evaluation of severe venous stasis. J Vasc Surg. 1994;20:721-7.

26. Criado E, Farber MA, Marston WA, Daniel PF, Burnham CB, Keagy BA. The role of air plethysmography in the diagnosis of chronic venous insufficiency. J Vasc Surg. 1998;27:660-70.

27. Oliveira RA, Barros Jr. N, Miranda Júnior F. A variabilidade hemodinâmica venosa detectada pelos parâmetros da pletismografia a ar nas classes clínicas da classificação CEAP. J Vasc Bras. 2007;6:4:359-65.

28. Allan JC. Volume changes in the lower limb in response to postural alterations and muscular exercises. S Afr J Surg. 1964;2:75-90.

29. Nicolaides AN. Diagnostic evaluation of patients with chronic venous insufficiency. In: Rutherford, RB. Vascular surgery. 3rd ed. Philadelphia: WB Saunders; 1989. p. 1583.

30. Christopoulos D, Nicolaides AN. Air plethysmography. In: Raju S, Villavicencio JL. Surgical management of venous disease. Baltimore: Williams \& Wilkins; 1997. p. 86-96.

31. Christopoulos D, Nicolaides AN, Galloway JM, Wilkinson A. Objective noninvasive evaluation of venous surgical results. J Vasc Surg. 1988;8:683-7.

32. Gillespie DL, Cordts PR, Hartono C, et al. The role of air plethysmography in monitoring results of venous surgery. $\mathrm{J}$ Vasc Surg. 1992;16:674-8.

33. Jiang P, van Rij AM, Christie RA, Hill GB, Thomson IA. Venous physiology in the different patterns of recurrent varicose veins and the relationship to clinical severity. Cardiovasc Surg. 2000;8:130-6. 
34. Owens LV, Farber MA, Yong ML, et al. The value of air plethysmography in predicting clinical outcome after surgical treatment of chronic venous insufficiency. J Vasc Surg. 2000;32:961-8.

35. Nishibe T, Nishibe M, Kudo F, Flores J, Miyazaki K, Yasuda K. Stripping operation with preservation of the calf saphenous veins for primary varicose veins: hemodynamic evaluation. Cardiovasc Surg. 2003;11:341-5.

36. van Rij AM, Jiang P, Solomon C, Christie RA, Hill GB. Recurrence after varicose vein surgery: a prospective long-term clinical study with duplex ultrasound scanning and air plethysmography. J Vasc Surg. 2003;38;935-43.

37. Kim IH, Joh JH. Kim DI. Venous hemodynamic changes in the surgical treatment of primary varicose vein of the lower limbs. Yonsei Med J. 2004;45:577-83.
38. Ting AC, Cheng SW, Wu LL, Cheung GC. Changes in venous hemodynamics after superficial vein surgery for mixed superficial and deep venous insufficiency. World J Surg. 2001;25:122-5.

\section{Correspondência:}

Prof. Dr. Nei R. A. Dezotti

Departamento de Cirurgia e Anatomia

FMRP-USP - Divisão de Cirurgia Vascular

Bandeirantes, 3900

CEP 14048-900 - Ribeirão Preto, SP

Tel.: (16) 3602.2593, (16) 3602.2407

Fax: (16) 3633.0836

E-mail: neidezotti@yahoo.com.br

\section{Colega Associado da SBACV}

Você está convidado a participar do crescimento e consolidação do J Vasc Bras - como autor, leitor ou revisor.

Leia e divulgue; conheça as normas e submeta seus trabalhos.

Jornal Vascular Brasileiro - Secretarial Editorial

Rua Maraguape, 72, loja 01

CEP 90690-380 - Porto Alegre - RS - Fone (51) 3012-0575

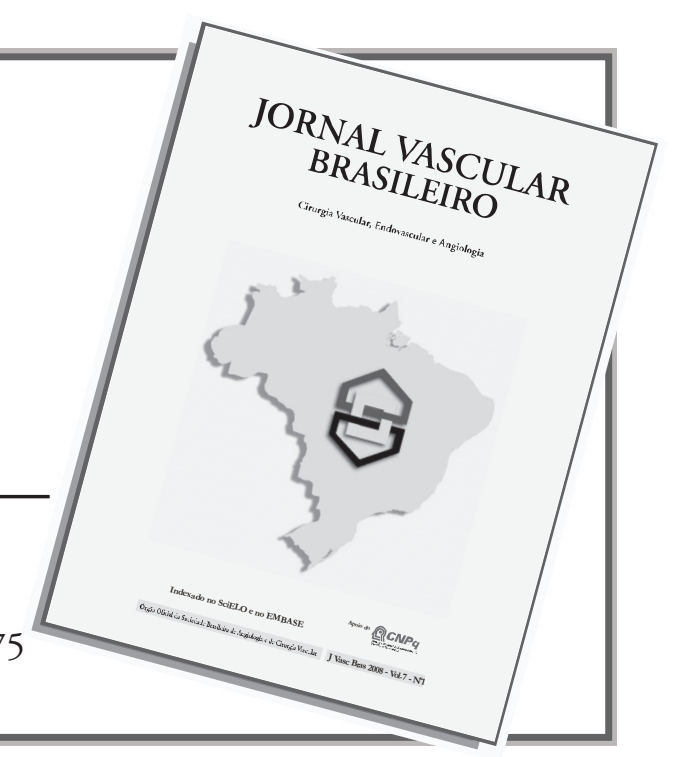

